\title{
Down with(out) neurogenesis
}

The function of adult neurogenesis is a topic of much research and debate. Reduced adult hippocampal neurogenesis has been associated with depression, but this link has so far been indirect, based mainly on findings that antidepressant drugs require neurogenesis to decrease depression-like behaviour in rodent models. Now, Snyder et al. provide more direct evidence that reduced adult hippocampal neurogenesis has a role in the aetiology of depression.

The authors created transgenic mice in which herpes simplex virus thymidine kinase was expressed under control of the glial fibrillary acidic protein (GFAP) promoter. Cells expressing GFAP, including neuronal precursor cells, were thereby made sensitive to the antiviral drug valganciclovir, which inhibits cell proliferation. Valganciclovir treatment in adult transgenic mice (v-TK mice) almost completely abolished hippocampal neurogenesis.

The authors first tested the reactivity of the hypothalamus-pituitary-adrenal (HPA) axis to stress in v-TK animals. Stress is thought to be an important factor in the aetiology of depression; indeed, chronic stress exposure is often used as an animal model of depression. Baseline plasma corticosterone levels and levels that were measured after exposure to a mild stressor - a new cage - were similar in control and v-TK mice. By contrast, following a single or chronic exposure to restraint, a stronger stressor, corticosteroid levels remained elevated for longer in $\mathrm{v}$-TK mice compared with control mice, indicating reduced negative feedback regulation of the HPA axis. In addition, although the synthetic corticosteroid dexamethasone could suppress restraintinduced corticosterone levels in v-TK mice, it did so to a lesser extent than in control mice. Notably, reduced dexamethasone suppression of corticosteroid release is also found in a proportion of people with depression.

On the basis of these results, the authors assessed the behaviour of $\mathrm{v}$-TK mice in paradigms that evoke varying levels of stress. In an openfield feeding test, control and v-TK animals behaved similarly, but if it was preceded by restraint stress, v-TK mice took longer to start eating. In the more stressful forced-swim test, $\mathrm{v}$-TK mice became immobile (a state that is assumed to signal behavioural 'despair') more quickly and remained so for longer. Lastly, in a sucrose preference test, which is thought to assess anhedonia (a common symptom of human depression), control and v-TK animals showed the same preference for sucrose, but when given the choice between the two drinks after a period of fluid deprivation $\mathrm{v}$-TK animals showed no sucrose preference.

These results show that eliminating adult hippocampal neurogenesis induces a depression-like behavioural and endocrinological phenotype in mice in stressful contexts. Some important questions remain to be addressed, such as the nature of the impaired feedback regulation and its relationship with the behavioural phenotype. Nevertheless, as previous studies have shown that high corticosteroid levels strongly suppress adult neurogenesis, the finding that corticosterone levels remain elevated for longer following stress exposure in $\mathrm{v}$-TK mice suggests a vicious cycle that might ultimately lead to depression.

Leonie Welberg

ORIGINAL RESEARCH PAPER Snyder, J. S. et al. Adult hippocampal neurogenesis buffers stress responses and depressive behaviour. Nature 3 Aug 2011 (doi: 10.1038/nature10287)

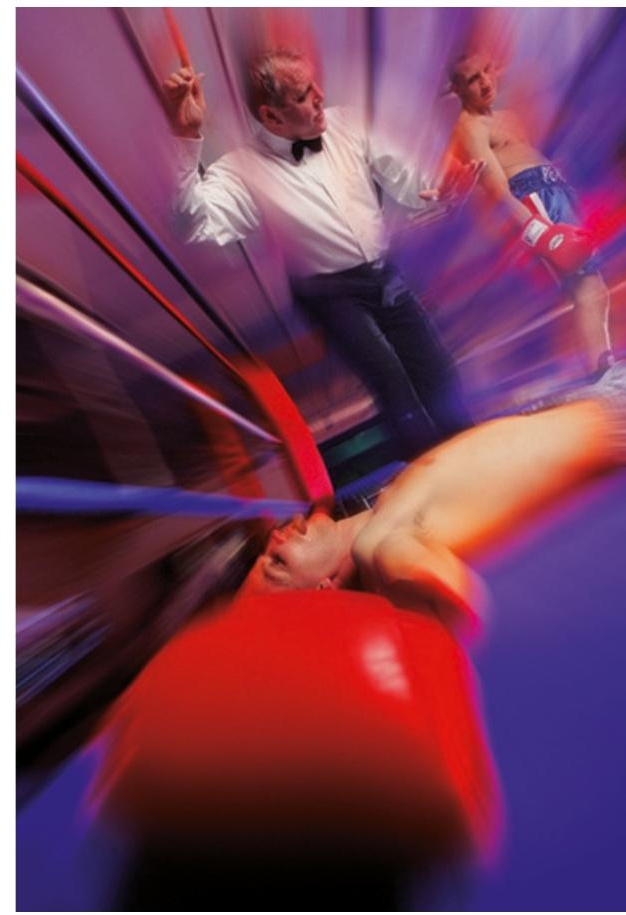

